\title{
PROGNOSTIC ACCURACY OF THE PROVENTRICULUS: KEEL RATIO FOR SHORT-TERM SURVIVAL IN PSITTACINES WITH PROVENTRICULAR DISEASE
}

\author{
Sophie E. Dennison, William M. Adams, Philippa J. Johnson, Brian S. Yandell, Joanne R. Paul-Murphy
}

\begin{abstract}
The proventriculus:keel ratio was evaluated as a prognostic indicator for short-term survival in parrots with proventricular disease. Measurements were made from right lateral radiographs of 41 parrots with proventricular disease. Results were compared with the previously described abnormal proventriculus:keel ratio range and to short-term clinical outcome at 1 -month postdiagnosis. The ratio in all parrots was $>0.52$, and $39 \%$ of parrots succumbed to their disease within 1 month of diagnosis. There was no significant association between the proventriculus:keel ratio and survival duration $(P=0.9)$. The proventriculus:keel ratio is a sensitive indicator of proventricular disease in parrots but is insensitive as a prognostic indicator. Veterinary Radiology \& Ultrasound, Vol. 50, No. 5, 2009, pp 483-486.
\end{abstract}

Key words: ileus, neuropathic gastric dilatation, parrot, proventriculitis, radiography.

\section{Introduction}

$\mathrm{P}$ ROVENTRICULAR DISEASE SHOULD be suspected in parrots exhibiting regurgitation, vomiting, anorexia, or defecation of undigested food. Causes include infectious to toxic proventriculitis, neuropathic gastric dilatation, mechanical ileus, and functional ileus. These causes commonly result in proventricular enlargement by hypertrophy or dilatation. ${ }^{1-8}$ Proventricular disease may be progressive and fatal without timely medical or surgical intervention. ${ }^{1-3}$ Neuropathic gastric dilatation, which is attributed to an avian Borna virus infection, ${ }^{9}$ is considered infectious and parrots frequently fail to respond to therapy. However, variable clinical improvement following the use of the COX-2 inhibitor celecoxib have been described. ${ }^{10}$

Recently we described the proventriculus:keel ratio as a highly sensitive and specific objective method for determining normal vs. abnormal proventriculus size in psittacines. A ratio $>0.52$ was associated with clinical proventricular disease. ${ }^{11}$ In that study, there were too few parrots with known proventricular disease to investigate any association between the magnitude of the ratio and prognosis. The purpose of the present study was to characterize such an association by comparing the proventriculus:keel ratios in proventricular disease to short-term outcome.

From the Department of Surgical Sciences (Dennison, Adams and Paul-Murphy) and the Department of Statistics and the Department of Horticulture (Yandell), School of Veterinary Medicine, University of Wisconsin, Madison, WI 53706; Great Western Referrals, Unit 10 County Park, Shrivenham Road, Swindon, Wiltshire SN1 2NT, UK (Johnson). Address correspondence and reprint requests to Sophie E. Dennison, at the above address. E-mail: dennison@svm.vetmed.wisc.edu

Received January 5, 2009; accepted for publication February 23, 2009. doi: 10.1111/j.1740-8261.2009.01582.x

\section{Materials and Methods}

Medical records were reviewed at a university veterinary medical teaching hospital and two privately owned veterinary practices to identify adult parrots with confirmed proventricular disease per necropsy, surgery, biopsy, fecal smear or culture, blood or serum concentrations of heavy metals, and serum ionized calcium levels. Definitive diagnosis, method of confirmation and outcome, either terminal disease or survival, were recorded from the medical records. Terminal disease was death directly attributable to the proventricular disease. Survival was recorded if the clinical signs resolved or the parrot survived at least 1 month following presentation.

Only parrots with whole body right lateral radiographs acquired under general anesthesia, following at least $1 \mathrm{~h}$ of fasting were considered for inclusion; visualization of the margin of the proventriculus was an inclusion criterion. Additional radiographic criteria required inclusion of the entire coelom, spine, and keel of the sternum in a right lateral view, as well as good positioning and diagnostic technique. Acceptable positioning required partial to full superimposition of coracoid bones, as well as coxofemoral joint alignment within a width of the femoral head. The proventriculus:keel ratio was calculated for each parrot from the right lateral radiograph using a previously described method. ${ }^{11}$ The diameter of the proventriculus was measured from serosa to serosa, perpendicular to the long axis of the proventriculus, at the level of the thoracosynsacral junction. The height of the keel of the sternum was measured immediately caudal to the superimposition of the distal margins of the coracoid bones on the sternum, perpendicular to the dorsal margin of the sternum. The ratio was calculated by dividing the proventriculus measurement 


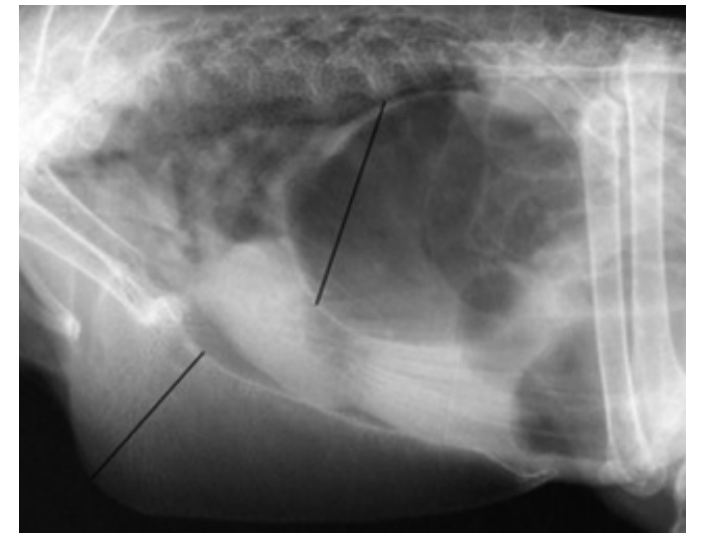

FIG. 1. Radiograph of a cockatoo with a linear foreign body causing obstruction of the proventriculus, ventriculus, and duodenum. Note marked gas distention of all three organs, consistent with complete or nearly complete obstruction. The foreign body was removed successfully during surgery. The black lines represent the measurements used for quantification of the proventriculus:keel ratio. In this parrot the calculated ratio was 1.37 (normal is $<0.48^{11}$ ).

by the keel measurement (Fig. 1). A ratio $<0.48$ is within normal limits, while a ratio $>0.52$ is consistent with proventricular disease, as described previously. ${ }^{11}$

Forty-one adult parrots presented between 2004 and 2007 and fulfilled the inclusion criteria (Table 1). Data were grouped into terminal disease and survival for statistical analysis, and proventriculus:keel ratios for these two groups were compared using Wilcoxon's rank-sum test for nonparametric data.

\section{Results}

The proventriculus:keel ratio was $>0.52$ in all 41 parrots, consistent with proventricular disease. ${ }^{11}$ The radiographic appearance of the enlarged proventriculus ranged from uniform soft tissue opacity, to varying proportions of intraluminal gas and soft tissue in combination, to gas

Table 1. Proventricular Disease Distribution by Common Parrot Name

\begin{tabular}{|c|c|c|c|c|}
\hline & $\begin{array}{l}\text { Proventriculitis } \\
\qquad(N=21)\end{array}$ & $\begin{array}{c}\text { Functional } \\
\text { Ileus } \\
(N=7)\end{array}$ & $\begin{array}{c}\text { Neuropathic } \\
\text { Gastric Dilatation } \\
(N=7)\end{array}$ & $\begin{array}{c}\text { Mechanical } \\
\text { Ileus } \\
(N=6)\end{array}$ \\
\hline African gray & 6 & 3 & 1 & \\
\hline Amazon & 4 & 1 & & 2 \\
\hline Budgerigar & 2 & & & \\
\hline Cockatoo & 2 & & 1 & 3 \\
\hline Conure & 1 & 1 & & \\
\hline Eclectus & & 1 & 1 & \\
\hline Lovebird & 1 & & & \\
\hline Macaw & 4 & 1 & 4 & 1 \\
\hline Quaker & 1 & & & \\
\hline
\end{tabular}

Mechanical ileus indicates obstructive disease and includes foreign body ingestion and neoplasia. Functional ileus includes heavy metal intoxication, peritonitis and hypocalcemia. Proventriculitis includes infectious (bacterial and fungal) and aseptic inflammatory etiologies. distension only (Fig. 1). Intraluminal proventricular gas was evident in $26 / 41$ parrots $(63 \%)$.

Final diagnoses for the 41 parrots were proventriculitis $(21 / 41,51 \%)$, neuropathic gastric dilatation $(7 / 41,17 \%)$, functional ileus $(7 / 41,17 \%)$, and mechanical ileus $(6 / 41$, $15 \%$ ) (Table 1). Diagnosis of proventriculitis was recorded from necropsy samples $(3 / 21)$, crop biopsy $(6 / 21)$, proventricular biopsy $(2 / 21)$ or from an abnormal fecal swab smear/culture accompanied by appropriate clinical signs, and a corresponding inflammatory leukogram (10/21). In the six parrots that had crop biopsies performed, inflammatory changes were identified without evidence of ganglioneuritis, and this finding with a radiographically abnormal proventriculus led to a presumptive diagnosis of ingluvitis with proventriculitis. Abnormal fecal swabs included evidence of fungal infection on fecal smear $(7 / 10)$, or abnormally heavy bacterial growth on culture $(3 / 10)$. Neuropathic gastric dilatation diagnosis was made following histologic identification of characteristic lymphoplasmacytic ganglioneuritis ${ }^{12}$ from crop biopsy (2/7) or necropsy samples of proventriculus or brain (5/7). Functional ileus was diagnosed from elevated heavy metal concentrations in blood (lead, 4/7) or serum (zinc, 1/7), marked hypocalcemia $(1 / 7)$, or by identification of peritonitis at necropsy (1/7). Mechanical ileus was diagnosed surgically (5/6) as foreign body ingestion and at necropsy $(1 / 6)$ as adenocarcinoma.

Of the 41 parrots, 16 died or were euthanized due to their proventricular disease, within 1 month of imaging. Terminal disease was identified in all groups; $3 / 21$ parrots with proventriculitis, $7 / 7$ parrots with neuropathic gastric dilatation, 6/7 parrots with functional ileus, and 1/6 parrots with mechanical ileus. The three parrots with proventriculitis that died included two with infectious proventriculitis attributed to Macrorhabdus ornithogaster and one with aseptic inflammatory proventriculitis combined with esophagitis, ingluvitis, ventriculitis, and proximal enteritis.

Twenty-five parrots were alive and without clinical signs 1 month after diagnosis. No significant difference in proventriculus:keel ratio was found in the short-term between the terminal disease group and the surviving group (Fig. 2, Table 2; $P=0.9)$.

\section{Discussion}

The previously reported abnormal proventriculus:keel ratio threshold of $0.52^{11}$ was verified in the 41 parrots in this study. There was no association between the magnitude of the abnormal proventriculus:keel ratio and 1-month survival. While the number of parrots in each disease group is low, overlap of the proventriculus:keel ratio between survivor and terminal disease groups precludes use of this ratio as a prognostic indicator. 


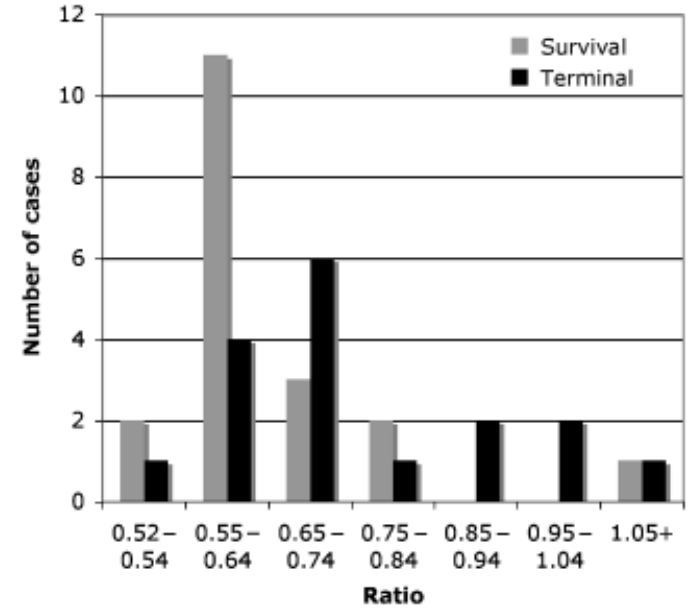

Fig. 2. Histogram comparing terminal disease and survival across the measured range of proventriculus:keel ratios calculated for parrots with proventricular disease. There was no significant difference between the ratios calculated from parrots with terminal disease vs. those that survived 1-month postdiagnosis $(P=0.9)$.

Parrots in the terminal disease group included both small and large psittacines and all four disease processes, consistent with many proventricular diseases being potentially fatal. However, $61 \%$ of psittacines had at least shortterm recovery from their proventricular disease.

Proventriculitis was the most frequently diagnosed condition, affecting 21 parrots or $51 \%$ of the cohort. Both aseptic inflammatory and infectious causes were recorded. The number of parrots in this group is not unsurprising given the number of infectious and aseptic etiologies that fall under this category. Only $3 / 21$ parrots $(14 \%)$ died as a direct result of proventriculitis. This was probably due to the high proportion of parrots that were infectious and could be treated with antimicrobial drugs. It is possible, using the methods of diagnosis described herein, that more parrots with infectious proventriculitis were misdiagnosed as aseptic proventriculitis due to failure to identify the organism. As antimicrobial drugs were commonly recommended prophylactically in the aseptic proventriculus group to prevent secondary infection, it is difficult to know if misdiagnoses were made. The two budgerigars with $M$. ornithogaster succumbed to the infection. This yeast-like organism is difficult to treat and can result in

TABLE 2. Short-Term Outcome by Proventricular Disease Process

\begin{tabular}{lcc}
\hline Disease Process & Terminal Disease & Survival \\
\hline Neuropathic gastric dilatation & 7 & 0 \\
Functional ileus & 6 & $1^{\wedge}$ \\
Proventriculitis & 3 & 18 \\
Mechanical ileus & $1^{*}$ & 5 \\
\hline
\end{tabular}

Mechanical ileus indicates obstructive disease and includes neoplasia $\left(^{*}\right)$ and foreign body ingestion. Functional ileus includes heavy metal intoxication, peritonitis, and hypocalcemia $\left({ }^{\wedge}\right)$. Proventriculitis includes infectious (bacterial and fungal) and aseptic inflammatory etiologies. severe, acute proventriculitis with acute death. Conversely, a chronic form of the disease characterized by progressive weight loss has also been described in budgerigars and other avian species. ${ }^{6}$

Neuropathic gastric dilatation was the second most common disease diagnosed, accounting for seven parrots in the cohort. All parrots with neuropathic gastric dilatation succumbed to the disease. This diagnosis was common in macaws, as suggested in the literature, accounting for four of seven subjects $(57 \%)$. Even so, the overall incidence of neuropathic gastric dilatation within this population was $17 \%$, much lower than the suggested prevalence. ${ }^{1-3,7-9}$ Histopathologic identification of characteristic lymphoplasmacytic ganglioneuritis from crop biopsy samples is currently the gold standard diagnostic test for neuropathic gastric dilatation, ${ }^{12}$ however, if representative lesions are not sampled, false negatives may result. Thus the true number of parrots with neuropathic gastric dilatation may have been higher than those identified. As long-term follow up was not available, a limitation of this study is the possibility of underlying neuropathic gastric dilatation in parrots that responded to symptomatic therapy for a month.

Functional ileus was identified in seven parrots and attributed to heavy metal intoxication in $5 / 7(71 \%)$, hypocalcemia in $1 / 7(14 \%)$, and peritonitis in $1 / 7(14 \%)$. The presumptive mechanism of proventricular enlargement in functional ileus is dilatation secondary to decreased motility and lack of muscular tone. Of particular interest is the solitary African gray parrot with hypocalcemic-associated functional ileus; with resolution of clinical signs and normalization of serum ionized calcium levels the proventriculus:keel ratio returned to within normal limits.

An unexpected finding was proventricular disease associated with mechanical ileus that was attributed to foreign body ingestion in $5 / 6$ parrots $(83 \%)$ and adenocarcinoma involving the proventriculus in $1 / 6(17 \%)$. Foreign body location was identified as proventriculus alone in $3 / 5$ $(60 \%)$, proventriculus and ventriculus in $1 / 5(20 \%)$, and proventriculus, ventriculus, and duodenum in $1 / 5(20 \%)$. Interestingly, $3 / 5(60 \%)$ of parrots with foreign body ingestion were young adult cockatoos, which is similar to a previous report. ${ }^{4}$ All foreign bodies were successfully removed with subsequent recovery. Neoplasia of the psittacine gastrointestinal tract is an unusual cause for mechanical obstruction. ${ }^{1,5}$

Intraluminal proventricular gas was identified in $63 \%$ of radiographs. This may have been due to the underlying disease, or to aerophagia associated with anesthesia. Association with recent feeding is less likely as only radiographs of parrots that were fasted for at least $1 \mathrm{~h}$ before anesthesia and radiography were included. In our previous study, gas within the proventriculus was also recognized in both healthy parrots and in parrots with proventricular disease. This did not result in miscategorization of any healthy par- 
rot as diseased or vice versa, ${ }^{11}$ however, any gas introduced into the proventriculus in association with anesthesia may affect the magnitude of an abnormal ratio. This provides additional explanation why the magnitude of the proventriculus:keel ratio is an insensitive prognostic indicator.

The proventriculus:keel ratio cannot be measured accurately in all parrots due to effacement of the ventral proventricular serosal margin. This may occur when the proventriculus lies in direct contact with the liver in birds with hepatomegaly or when marked proventricular enlargement occurs without the presence of intraluminal gas. While the presence of intraluminal proventricular gas will only delineate the mucosal surface of the proventriculus, this will assist in the identification of the serosal margin in many cases. Border effacement also occurs in the presence of coelomic effusion. With increased availability of digital radiography, it is anticipated that the fraction of parrots wherein the measurement cannot be made will decrease.

In conclusion, the proventriculus:keel ratio measured from whole body right lateral radiographs consistently allowed identification of proventricular enlargement associated with proventricular disease, but there was no association between the magnitude of the ratio and prognosis for short-term survival.

\section{REFERENCES}

1. Gelis S. Evaluating and treating the gastrointestinal system. In: Harrison GJ, Lightfoot TL. (eds): Clinical avian medicine. Florida: Spix Publishing Inc., 2006;429-432.

2. Schmidt RE. Pathology of gastrointestinal disease in psittacine parrots. Sem Avian Exot Pet Med 1999;8:75-82.

3. Hadley TL. Disorders of the psittacine gastrointestinal tract. Vet Clin Exot Anim 2005;8:329-349.

4. Adamcak A, Hess LR, Quesenberry KE. Intestinal string foreign body in an Umbrella Cockatoo (Cacatua alba). J Avian Med Surg 2000;14: 257-263.

5. Leach MW, Paul-Murphy J, Lowenstine LJ. Three cases of gastric neoplasia in psittacines. Avian Dis 1989;33:204-210.

6. Phalen D. Diagnosis and management of Macrorhabdus ornithogaster (formerly Megabacteria). Vet Clin N Am:Exot Anim Pract 2005;8:299-306.

7. Mannl A, Gerlach H, Leipold R. Neuropathic gastric dilatation in psittaciformes. Avian Dis 1987;31:214-221.
8. Dustan Clark F. Proventricular dilatation syndrome in large psittacine parrots. Avian Dis 1984;28:813-815.

9. Kistler AL, Gancz A, Clubb S, et al. Recovery of divergent avian bornaviruses from cases of proventricular dilatation disease: identification of a candidate etiologic agent. Virol J 2008;5:88-102.

10. Dahlhausen B. Resolution of clinical proventricular dilatation disease by cyclooxygenase 2 inhibition. (Abstract). Proc Annual Conf Assoc Avian Vet 2002;9-12.

11. Dennison SE, Paul-Murphy JR, Adams WM. Radiographic determination of proventricular diameter in psittacine parrots. J Am Vet Med Assoc 2008;232:709-714.

12. Gregory RC, Latimer KS, Campagnoli RP, Ritchie BW. Histologic evaluation of the crop for diagnosis of proventricular dilatation syndrome in psittacine parrots. J Vet Diagn Invest 1996;8:76-80. 